\title{
Better Solar Cells and Manufacturing Processes Using NREL's Ultrafast Quantum Efficiency Method
}

\section{A solid-state optical system by NREL and Tau Science measures solar cell quantum efficiency in less than a second, enabling a suite of new capabilities for solar cell manufacturers.}

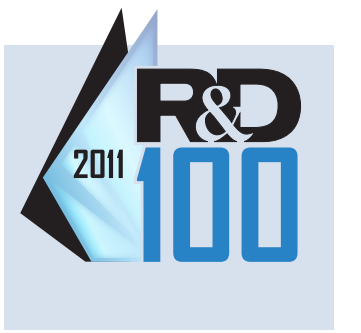

\section{Houston, We Have a Problem...}

Quantum efficiency (QE) measurements indicate how well a solar cell converts the various wavelengths of sunlight into electricity. Optimally, a solar cell should generate considerable electrical current for wavelengths that are most abundant in sunlight. And the ability to measure QE provides a crucial diagnostic tool for solar cell performance.

Manufacturers of solar cells would like to be able to measure the QE of every cell moving through their production lines. But there's a problem: an individual cell moves past a reference point about every second, but a $\mathrm{QE}$ measurement using the conventional process takes up to 20 minutes. Clearly, only a very small number of cells can be measured as representatives of a vastly larger batch whizzing through the production line.

\section{Doing Things in Parallel}

The crux of the measurement malaise is serial processing. That is, the conventional technique's approach is to step through a range of frequencies of light, measuring one data point at a time until the entire QE curve (see sidebar on reverse) is produced across this range.

However, scientists at the National Renewable Energy Laboratory (NREL) had a eureka moment when considering the possibility of using parallel processing to speed things up. They actually combined three key components to allow entire QE curves to be generated within one second - or more than 1,000 times faster than the industry's state of the art.

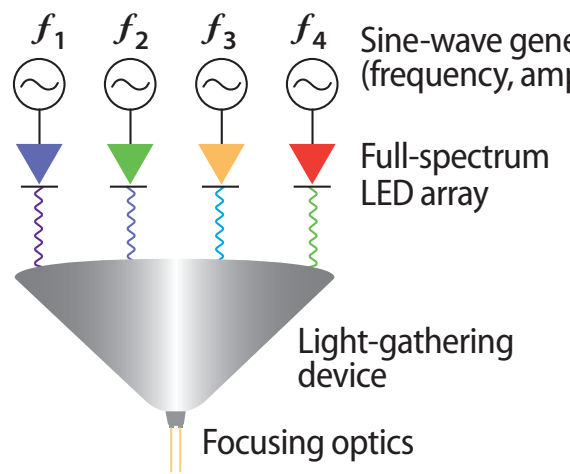

\begin{tabular}{|l|c|c|}
\hline $\begin{array}{c}\text { LED } \\
\text { (\#, color) }\end{array}$ & $\begin{array}{c}\lambda \text {-peak } \\
\text { (nm) }\end{array}$ & $\begin{array}{c}\text { Blinking } \\
\text { frequency } \\
\text { (Hz) }\end{array}$ \\
\hline 1. Blue & 470 & 875 \\
\hline 2. Green & 550 & 1262 \\
\hline 3. Yellow & 600 & 1153 \\
\hline 4. Red & 700 & 1000 \\
\hline
\end{tabular}

Simplified schematic of the principle behind the LED light sources being synchronized electronically to sort out the electrical responses. Each LED is driven by a different "blinking" frequency that uniquely links cell response to a specific color of LED, thus allowing parallel processing.
An array of light-emitting diodes (LEDs) is at the heart of NREL's ultrafast measurement approach, expanded and commercialized by Tau Science as the FlashQE ${ }^{\mathrm{TM}}$ system. Each of several dozen different-colored LEDs is driven on and off ("blinked") and encoded by a unique frequency.

Using a signal-conditioning assembly, the solar cell's electrical response to light from an individual LED can be tied to the unique driving frequency. Thus, this clever design allows the light from all the LEDs to shine simultaneously on the cell, and the individual electrical responses can be teased out because each is driven by a unique frequency that can be traced back to the specificcolor LED. This parallel processing allows multiple QE measurements to be made at once, rather than one after another as in the one-point-at-a-time conventional approach.

Special mathematical techniques, involving Fast Fourier Transform analysis, are the third component coupled with the LEDs and signal-conditioning system. This technique can look at an apparent jumble of data coming in simultaneously and quickly sort it out, assigning responses to the specific spectrum of light shining on the solar cell and generating the complete QE curve.

\section{A Trove of New Capabilities}

The FlashQE system's extremely rapid QE measurements are not some incremental improvement over current options. Rather, its 1,000x increase in measurement

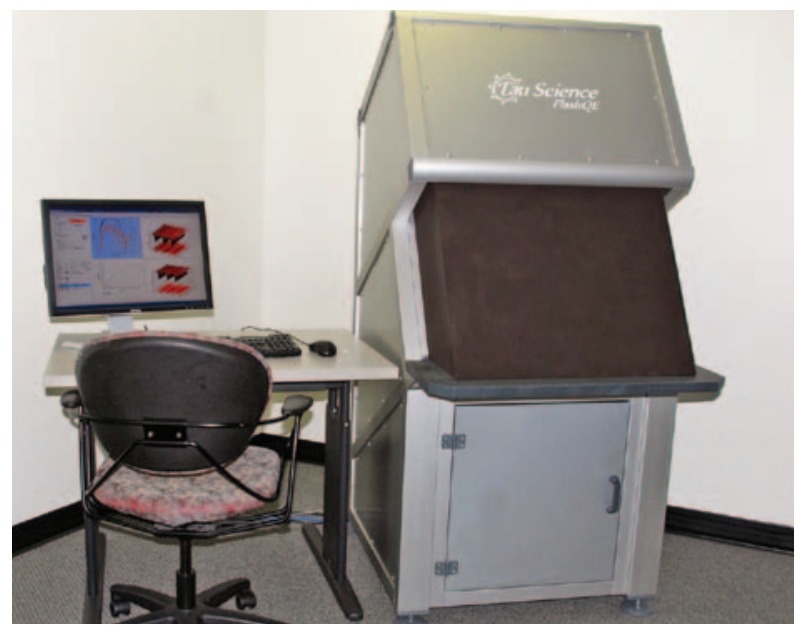

Photo from Jamie Hudson, Tau Science

Tau Science's commercial FlashQE system based on NREL's realtime measurement approach. When placed within the unit on the right, the cell is illuminated and measured to produce $a \mathrm{QE}$ curve that appears nearly instantly on the monitor. 
speed is enabling numerous new capabilities for industry and laboratory researchers. For example, the system can:

- Serve as an in-line tool within the solar cell manufacturing operation because measurement times are in sync with the rate of moving cells through the line.

- Measure every wafer within the processing batch, rather than taking one representative sample in every 10,000 or 100,000 samples.

- Monitor the quality of the entire manufacturing process; that is, the QE of each wafer can be compared to the ideal QE of what the manufacturer expects. If there are unacceptable deviations, production can be stopped quickly, and processing equipment upstream can be checked and adjusted.

- "Bin" wafers by QE, which means that wafers with similar QE characteristics can be identified and kept together. Modules made from cells in the same bins lead to optimal performance of the completed modules.

- Map entire wafers, mostly within a laboratory setting. The speed of FlashQE allows researchers to take numerous measurements across a solar cell and compile them into a 3-D map. Such mapping can help to understand underlying causes of performance inhomogeneity within regions of a wafer and detect material problems within the cell.

In addition to these capabilities, the operational and maintenance costs for the FlashQE system are less than for conventional mechanically driven spectrometer systems.

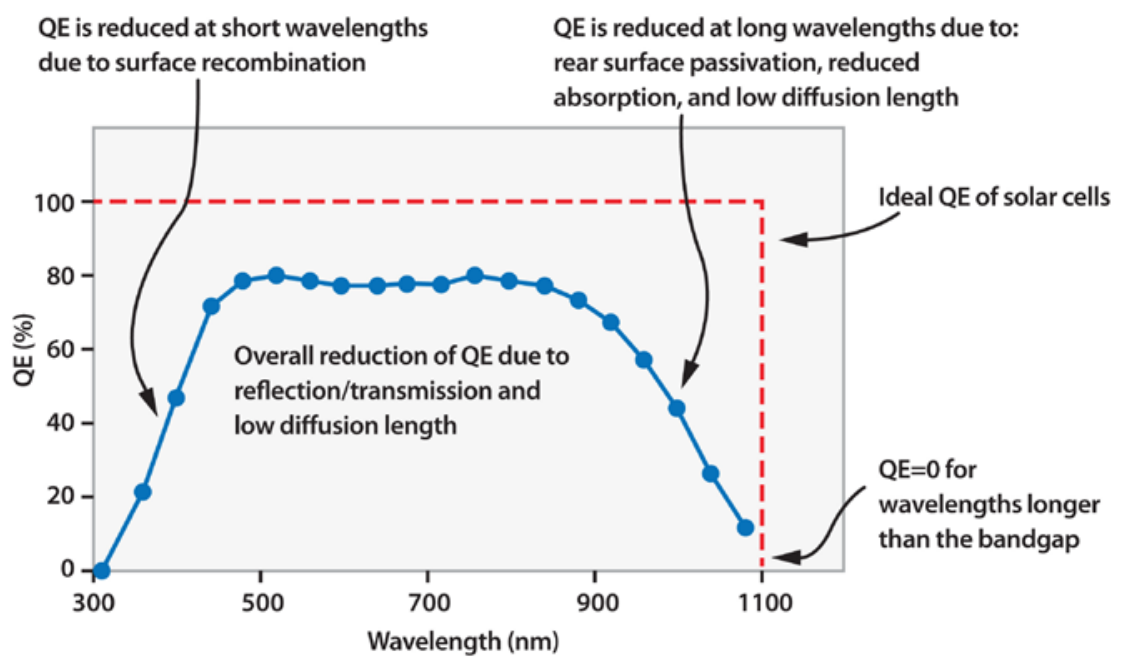

This basic QE plot for a silicon solar cell shows percent QE vs. wavelength of light. The annotations indicate generic changes in the QE curve shape that depend on particular characteristics of the cell being measured.
And its unsurpassed precision-virtually identical QE curves for the same sample measured 32,000 times over a 9-hour period-highlights the stability of the new system.

\section{A New Industry Standard}

The FlashQE system truly is an elegant solution to the problematic slow-as-molasses measurements of the conventional QE system. The synergistic coupling of solidstate light sources, electronic control and measurement, and mathematical analysis has produced an ultrafast system. But the true power of this blazing speed is its function in enabling totally new capabilities needed by the solar industry. Such capabilities lead to better solar cells and modules, improved manufacturing production, and increased energy production. And this NREL technique, now available commercially, is almost certain to become the new industry standard for QE measurements.

\section{So What Does QE Tell You?}

The shape of a QE curve helps scientists and manufacturers to understand various characteristics of a solar cell.

The curve will be low in the left-hand region (below) if it has poor spectral response to blue light-that is, the cell does not use shorterwavelength light effectively to produce electrical current. Conversely, the curve will be low in the right-hand region if it has poor spectral response to longer-wavelength red light. These deviations from the ideal indicate characteristics of the cell that need to be addressed-such as altering the material composition, thickness of layers, or quality of front or back surfaces.

The best actual cell will approach the ideal curve over the wavelengths of the sunlight's spectrum that the cell is able to absorb. Cells with good red response show optimal performance at dawn or dusk, whereas cells with good blue response generate the most energy when the sun is higher in the sky.

\section{For More Information}

NREL: David Young 303-384-6621

david.young@nrel.gov
Tau Science: James Hudson 503-828-1375 contact@tauscience.com 